\title{
Stokes Flow past a Swarm of Porous Nanocylindrical Particles Enclosing a Solid Core
}

\author{
Satya Deo and Pramod Kumar Yadav \\ Department of Mathematics, University of Allahabad, Allahabad 211002, India \\ Correspondence should be addressed to Satya Deo, satyadeo_verma@rediffmail.com
}

Received 21 May 2007; Revised 12 July 2007; Accepted 5 December 2007

Recommended by Manfred Moller

This paper concerns the Stokes flow of an incompressible viscous fluid past a swarm of porous nanocylindrical particles enclosing a solid cylindrical core with Kuwabara boundary condition. An aggregate of porous nanocylindrical particles is considered as a hydro-dynamically equivalent to a solid cylindrical core with concentric porous cylindrical shell. The Brinkman equation inside the porous cylindrical shell and the Stokes equation outside the porous cylindrical shell in their stream function formulations are used. Explicit expressions for the stream functions in both regions have been investigated. The drag force acting at each nanoporous cylindrical particle in a cell is evaluated. Also, we solved the same problem by using Happel boundary condition on the hypothetical cell. In certain limiting cases, drag force converges to pre-existing analytical results, such as the drag on a porous circular cylinder and the drag on a solid cylinder in Kuwabara's cell or Happel's cell. Representative results are then discussed and compared for both cases and presented in graphical form by using Mathematica software.

Copyright (C) 2008 S. Deo and P. K. Yadav. This is an open access article distributed under the Creative Commons Attribution License, which permits unrestricted use, distribution, and reproduction in any medium, provided the original work is properly cited.

\section{Introduction}

The classical problems of the motion of objects through fluids continue to be of interest because of their applications in physical sciences and chemical engineering. A variety of physical situations arises in which the size of moving objects varies from micro- $\left(10^{-6}\right.$ meter $)$ to nano- $\left(10^{-9}\right.$ meter) scales. The computational predictions of the relevant hydrodynamical parameters of the flow of a viscous incompressible fluid past a swarm of porous particles at nanoscale are of considerable practical and theoretical interest of many physical, engineering, and medical problems.

Happel [1,2] and Kuwabara [3] proposed a cell model in which two concentric cylinders/spheres serve as the model for fluid moving through an assemblage of circular cylinders/spheres. The Kuwabara model assumes uniform velocity condition and vanishing of vorticity at the cell surface, whereas, Happel model assumes vanishing of shear stress instead 
of vanishing of vorticity at the cell surface. An analytical study of the steady incompressible flow past a circular cylinder embedded in a porous medium based on the Brinkman model has been reported by Pop and Cheng [4]. The drag on flow past a cylinder with slip was also evaluated by Datta and Shukla [5]. The problem of Stokes flow through a swarm of spherical particles moving in arbitrary direction was studied by Dassios and Vafeas [6] by using 3D Happel model. Stokes flow past a swarm of porous circular cylinders with Happel and Kuwabara boundary conditions was discussed by Deo [7]. Recently, a new model for calculating specific resistance of aggregated colloidal cake layers in membrane filtration processes was discussed by Kim and Yuan [8].

In the present work, the problem of the Stokes flow past a swarm of porous nanocylindrical particles enclosing a solid cylindrical core with Kuwabara boundary condition is considered. The Brinkman equation for the flow inside and the Stokes equation outside the porous cylindrical shell in their stream function formulations is used. The drag force experienced by each nanoporous circular cylindrical particle in a cell is evaluated. Representative results are presented in graphical form by using Mathematica and they are compared in both cases. The Happel formulation is slightly superior because it leads to particles-in-cell that are selfsufficient in mechanical energy [9]. Special known results are then also deduced from the present analysis.

\section{Statement and mathematical formulation of the problem}

A primary assumption employed in this study is that a swarm of nanosized porous coaxial (along z-axis) cylindrical particles surrounding a solid cylindrical core having the same axis is hydro-dynamically equivalent to a coaxial porous cylindrical shell surrounding the solid core. Let the radius of the solid cylindrical core be $a$ and let the radius of the concentric porous cylindrical shell enclosing the solid cylindrical core be $b(b>a)$. Further, we assume that this porous shell is enveloped by a concentric cylinder of radius $c(c>b)$ named as cell surface, and let the radius of each nano porous cylindrical particle be $a_{p}$ (Figure 1). Also, we assume that the fluid is approaching towards the cell surface as well as partially passing through the composite cylinder perpendicular to the axis of cylinder (z-axis) with velocity $U$ from left to right. The radius $c$ of hypothetical cell is so chosen that the solid volume fraction $\gamma$ of the swarm is equal to the solid volume fraction of the cell, that is,

$$
\gamma=\frac{\pi b^{2}}{\pi c^{2}}
$$

\subsection{Governing equations}

The governing equation of incompressible Newtonian creeping flow for clear fluid, that is, outside the porous cylindrical shell, is governed by Stokes equation (Happel and Brenner [10])

$$
\mu_{1} \nabla^{2} \mathbf{v}^{(1)}=\nabla p^{(1)}
$$

Also, we assume that the flow inside the porous cylindrical shell is governed by Brinkman's [11] equation

$$
\nabla^{2} \mathbf{v}^{(2)}-\left(\frac{\sigma^{2}}{b^{2}}\right) \mathbf{v}^{(2)}=\left(\frac{1}{\mu_{2}}\right) \nabla p^{(2)}
$$




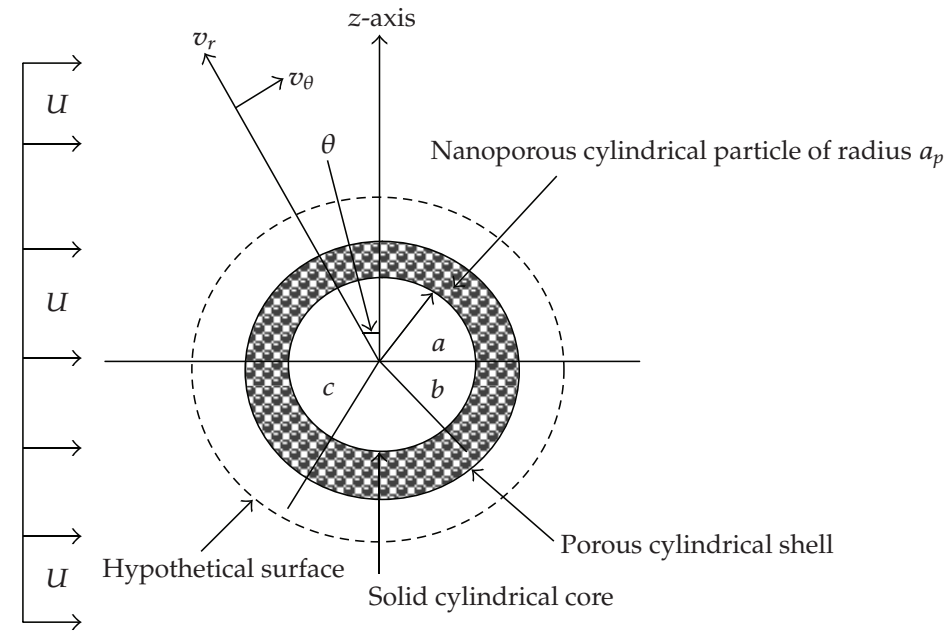

Figure 1: The physical model and the coordinate system.

Here, $\sigma^{2}=\beta b^{2} / k$, with $\beta=\mu_{1} / \mu_{2}, \mu_{1}$ is the viscosity of the fluid, $\mu_{2}$ denotes the effective viscosity of porous medium, with $k$ being the permeability of porous medium. Since, $\sigma$ is a dimensionless number related with the permeability, therefore we called it as dimensionless permeability parameter. Here, $\mathbf{v}^{(i)}, p^{(i)}, i=1,2$, are the velocity vector and pressure outside and inside the porous cylindrical shell, respectively.

The equations of continuity for axisymmetric, incompressible viscous fluid in cylindrical polar coordinates $(r, \theta, z)$ in both regions can be written as

$$
\frac{\partial}{\partial r}\left(r v_{r}^{(i)}\right)+\frac{\partial}{\partial \theta}\left(v_{\theta}^{(i)}\right)=0,
$$

where $v_{r}^{(i)}$ and $v_{\theta}^{(i)}, i=1,2$, are components of velocities in the direction of $r$ and $\theta$, respectively. The stream functions $\psi^{(i)}(r, \theta)$ in both regions satisfying equations of continuity (2.4) may be defined as

$$
v_{r}^{(i)}=\frac{1}{r} \frac{\partial \psi^{(i)}}{\partial \theta}, \quad v_{\theta}^{(i)}=-\frac{\partial \psi^{(i)}}{\partial r} .
$$

Therefore, on elimination of pressures in both (2.2) and (2.3) and on using (2.4), we get the following fourth-order partial differential equations, respectively, as

$$
\begin{gathered}
\nabla^{4} \psi^{(1)}=\nabla^{2}\left(\nabla^{2} \psi^{(1)}\right)=0, \\
\nabla^{4} \psi^{(2)}-\left(\frac{\sigma^{2}}{b^{2}}\right) \nabla^{2} \psi^{(2)}=\nabla^{2}\left(\nabla^{2}-\left(\frac{\sigma^{2}}{b^{2}}\right)\right) \psi^{(2)}=0,
\end{gathered}
$$

where the Laplacian operator

$$
\nabla^{2}=\frac{\partial^{2}}{\partial r^{2}}+\frac{1}{r} \frac{\partial}{\partial r}+\frac{1}{r^{2}} \frac{\partial^{2}}{\partial \theta^{2}}
$$

with the macroscopic assumption of $\mu_{1} / \mu_{2}=1$. 
The range of $r$ and $\theta$ in the above (2.6) and (2.7) within a cylinder can be given below as

$$
0<r<\infty, \quad 0<\theta \leq 2 \pi
$$

Furthermore, the expressions for tangential and normal stresses $T_{r \theta}^{(i)}, T_{r r}^{(i)}, i=1,2$, respectively, are given by

$$
\begin{aligned}
& T_{r \theta}^{(i)}=\mu_{i}\left[\frac{1}{r^{2}} \frac{\partial^{2} \psi^{(i)}}{\partial \theta^{2}}+\frac{1}{r} \frac{\partial \psi^{(i)}}{\partial r}-\frac{\partial^{2} \psi^{(i)}}{\partial r^{2}}\right], \\
& T_{r r}^{(i)}=-p^{(i)}+\frac{2 \mu_{i}}{r}\left[\frac{\partial^{2} \psi^{(i)}}{\partial r \partial \theta}-\frac{1}{r} \frac{\partial \psi^{(i)}}{\partial \theta}\right] .
\end{aligned}
$$

Also, the pressure may be obtained in both regions (Happel and Brenner [10]) by integrating the following relations, respectively, as

$$
\begin{gathered}
\frac{\partial p^{(i)}}{\partial r}=\mu_{i}\left[\nabla^{2} v_{r}^{(i)}-\frac{v_{r}^{(i)}}{r^{2}}-\frac{2}{r^{2}} \frac{\partial v_{\theta}^{(i)}}{\partial \theta}-\delta_{2 i}\left(\frac{\sigma}{b}\right)^{2} v_{r}^{(i)}\right], \\
\frac{1}{r} \frac{\partial p^{(i)}}{\partial \theta}=\mu_{i}\left[\nabla^{2} v_{\theta}^{(i)}-\frac{v_{\theta}^{(i)}}{r^{2}}+\frac{2}{r^{2}} \frac{\partial v_{r}^{(i)}}{\partial \theta}-\delta_{2 i}\left(\frac{\sigma}{b}\right)^{2} v_{\theta}^{(i)}\right],
\end{gathered}
$$

where $\delta_{21}=0$ and $\delta_{22}=1$.

A suitable stream function solution of the Stokes equation (2.6) can be expressed as

$$
\psi^{(1)}(r, \theta)=U b\left[A_{1} r^{\prime}+B_{1} r^{\prime 3}+\frac{C_{1}}{r^{\prime}}+D_{1} r^{\prime} \ln r^{\prime}\right] \sin \theta .
$$

A particular solution of the Brinkman equation (2.7) may be written as

$$
\psi^{(2)}(r, \theta)=U b\left[A_{2} r^{\prime}+\frac{B_{2}}{r^{\prime}}+C_{2} I_{1}\left(\sigma r^{\prime}\right)+D_{2} K_{1}\left(\sigma r^{\prime}\right)\right] \sin \theta
$$

Here, $I_{1}\left(\sigma r^{\prime}\right)$ and $K_{1}\left(\sigma r^{\prime}\right)$ are the modified Bessel functions of the order one of the first and second kinds (Abramowitz and Stegun [12]), respectively, and the dimensionless variable $r^{\prime}=$ $r / b$.

\section{Solution of the problem with Kuwabara boundary condition}

The boundary conditions, those are physically realistic and mathematically consistent for the problem, can be taken as given below. On the solid cylindrical core,

$$
v_{r}^{(2)}(a, \theta)=0, \quad v_{\theta}^{(2)}(a, \theta)=0
$$

On the porous surface,

$$
\begin{array}{ll}
v_{r}^{(2)}(b, \theta)=v_{r}^{(1)}(b, \theta), & v_{\theta}^{(2)}(b, \theta)=v_{\theta}^{(1)}(b, \theta), \\
T_{r r}^{(2)}(b, \theta)=T_{r r}^{(1)}(b, \theta), & T_{r \theta}^{(2)}(b, \theta)=T_{r \theta}^{(1)}(b, \theta) .
\end{array}
$$


On the hypothetical cell surface,

$$
v_{r}^{(1)}(c, \theta)=U \cos \theta
$$

The vanishing of vorticity on the cell surface, that is, Kuwabara condition, implies that

$$
\nabla^{2} \psi^{(1)}(c, \theta)=0 .
$$

\subsection{Determination of arbitrary constants}

Applying the boundary conditions given by (3.1)-(3.4) and solving the resulting equations, we get the values of all the arbitrary constants $A_{1}, B_{1}, C_{1}, D_{1}, A_{2}, B_{2}, C_{2}$, and $D_{2}$ appearing in (2.12) and (2.13).

\section{Evaluation of drag}

Integrating the normal and tangential stresses over the porous cylindrical shell of radius $b$ in a cell yields the experienced drag force $F$ per unit length as

$$
F=\int_{0}^{2 \pi}\left(T_{r r}^{(1)} \cos \theta-T_{r \theta}^{(1)} \sin \theta\right)_{r=b} r d \theta=4 \pi \mu_{1} U D_{1}
$$

where

$$
\begin{aligned}
D_{1}= & \frac{-4 m^{4} \sigma^{2}\left[2 I_{1}(\sigma \ell) K_{1}(\sigma)+\ell\left(1+\ell^{2}\right) \sigma I_{2}(\sigma \ell) K_{1}(\sigma)+I_{1}(\sigma)\left\{-2 K_{1}(\sigma \ell)+\ell\left(1+\ell^{2}\right) \sigma K_{2}(\sigma \ell)\right\}\right]}{\Delta}, \\
\Delta= & -16 m^{4} \ell \sigma I_{2}(\sigma \ell) K_{1}(\sigma)+8 \ell^{3} \sigma I_{2}(\sigma \ell) K_{1}(\sigma)+16 m^{2} \ell^{3} \sigma I_{2}(\sigma \ell) K_{1}(\sigma)-8 m^{4} \ell^{3} \sigma I_{2}(\sigma \ell) K_{1}(\sigma) \\
& +\ell \sigma^{3} I_{2}(\sigma \ell) K_{1}(\sigma)-4 m^{2} \ell \sigma^{3} I_{2}(\sigma \ell) K_{1}(\sigma)+3 m^{4} \ell \sigma^{3} I_{2}(\sigma \ell) K_{1}(\sigma)+\ell^{3} \sigma^{3} I_{2}(\sigma \ell) K_{1}(\sigma) \\
& -4 m^{2} \ell^{3} \sigma^{3} I_{2}(\sigma \ell) K_{1}(\sigma)+3 m^{4} \ell^{3} \sigma^{3} I_{2}(\sigma \ell) K_{1}(\sigma)+32 m^{4} I_{1}(\sigma) K_{1}(\sigma \ell)-2 \sigma^{2} I_{1}(\sigma) K_{1}(\sigma \ell) \\
& +8 m^{2} \sigma^{2} I_{1}(\sigma) K_{1}(\sigma \ell)-6 m^{4} \sigma^{2} I_{1}(\sigma) K_{1}(\sigma \ell)-8 \ell^{2} \sigma I_{2}(\sigma \ell) K_{1}(\sigma \ell)+8 m^{4} \ell^{2} \sigma I_{2}(\sigma \ell) K_{1}(\sigma \ell) \\
& -8 \ell \sigma I_{1}(\sigma) K_{2}(\sigma)+8 m^{4} \ell \sigma I_{1}(\sigma) K_{2}(\sigma)+4 \ell \sigma^{2} I_{2}(\sigma \ell) K_{2}(\sigma)-8 m^{2} \ell \sigma^{2} I_{2}(\sigma \ell) K_{2}(\sigma) \\
& +4 m^{4} \ell \sigma^{2} I_{2}(\sigma \ell) K_{2}(\sigma)+4 \ell^{3} \sigma^{2} I_{2}(\sigma \ell) K_{2}(\sigma)-8 m^{2} \ell^{3} \sigma^{2} I_{2}(\sigma \ell) K_{2}(\sigma)+4 m^{4} \ell^{3} \sigma^{2} I_{2}(\sigma \ell) K_{2}(\sigma) \\
& -16 m^{4} \ell \sigma I_{1}(\sigma) K_{2}(\sigma \ell)+8 \ell^{3} \sigma I_{1}(\sigma) K_{2}(\sigma \ell)+16 m^{2} \ell^{3} \sigma I_{1}(\sigma) K_{2}(\sigma \ell)-8 m^{4} \ell^{3} \sigma I_{1}(\sigma) K_{2}(\sigma \ell) \\
& +\ell \sigma^{3} I_{1}(\sigma) K_{2}(\sigma \ell)-4 m^{2} \ell \sigma^{3} I_{1}(\sigma) K_{2}(\sigma \ell)+3 m^{4} \ell \sigma^{3} I_{1}(\sigma) K_{2}(\sigma \ell) \\
& +\ell^{3} \sigma^{3} I_{1}(\sigma) K_{2}(\sigma \ell)-4 m^{2} \ell^{3} \sigma^{3} I_{1}(\sigma) K_{2}(\sigma \ell)+3 m^{4} \ell^{3} \sigma^{3} I_{1}(\sigma) K_{2}(\sigma \ell) \\
& +4\left(1-m^{2}\right) \sigma I_{2}(\sigma)\left[-2\left(1+m^{2}\right) \ell K_{1}(\sigma)-\left(-1+m^{2}\right)\left\{2 K_{1}(\sigma \ell)-\ell\left(1+\ell^{2}\right) \sigma K_{2}(\sigma \ell)\right\}\right] \\
& -4 m^{4} \ell \sigma^{3} I_{2}(\sigma \ell) K_{1}(\sigma) \ln m-4 m^{4} \ell^{3} \sigma^{3} I_{2}(\sigma \ell) K_{1}(\sigma) \ln m+8 m^{4} \sigma^{2} I_{1}(\sigma) K_{1}(\sigma \ell) \ln m \\
& -4 m^{4} \ell \sigma^{3} I_{1}(\sigma) K_{2}(\sigma \ell) \ln m-4 m^{4} \ell^{3} \sigma^{3} I_{1}(\sigma) K_{2}(\sigma \ell) \ln m \\
& +2 I_{1}(\sigma \ell)\left[4\left(-1+m^{2}\right) \sigma\left\{\left(-1+m^{2}\right) K_{2}(\sigma)+\left(1+m^{2}\right) \ell^{2} K_{2}(\sigma \ell)\right\}\right. \\
& \left.+K_{1}(\sigma)\left\{\sigma^{2}-4 m^{2} \sigma^{2}+m^{4}\left(-16+3 \sigma^{2}\right)-4 m^{4} \sigma^{2} \ln m\right\}\right] .
\end{aligned}
$$


Also, the drag coefficient $C_{D}$ can be defined as

$$
C_{D}=\frac{F}{(1 / 2) \rho U^{2} 2 b}=\frac{8 \pi D_{1}}{\operatorname{Re}},
$$

where $\operatorname{Re}=2 b U / \nu_{1}$ is the Reynolds number, and $\nu_{1}=\mu_{1} / \rho$ being the kinematic viscosity of fluid.

\subsection{Deductions of some known results}

\subsubsection{Drag on a porous circular cylinder in a cell}

If $a=0$, that is, $\ell=a / b=0$, then cylindrical shell will reduce to a porous circular cylinder of radius $b$. In this case, we get the value of the drag coefficient $C_{D}$ as

$$
C_{D}=\frac{32 \pi \sigma^{2} I_{1}(\sigma)}{\operatorname{Re}\left[\left\{-4\left(\sigma^{2}(1-\gamma)-4\right)+\sigma^{2}\left(-(2 \log \gamma+1)+\gamma^{2}\right)\right\} I_{1}(\sigma)+2 \sigma\left\{\sigma\left(1-\gamma^{2}\right) I_{1}(\sigma)+2(1-\gamma)^{2} I_{2}(\sigma)\right\}\right]}
$$

where $\gamma=\left(\pi b^{2}\right) /\left(\pi c^{2}\right)=1 / m^{2}$ being the particle volume fraction.

A known result has been reported earlier by Deo [7] for the drag force experienced by a porous circular cylinder in a cell.

\subsubsection{Drag on a solid cylinder in Kuwabara cell model $(k \rightarrow 0)$}

When permeability $k$ vanishes, that is, permeability parameter $\sigma \rightarrow \infty$, then the porous circular cylinder behaves like a solid cylinder of radius $b$. In this case, the value of the drag coefficient $C_{D}$ will become as

$$
C_{D}=\frac{32 \pi}{\operatorname{Re}\left(4 \gamma-\gamma^{2}-3-2 \ln \gamma\right)}
$$

A known result for the drag has been reported earlier by Kuwabara [3].

\subsubsection{Happel boundary condition}

Happel assumes that on the cell surface shear stress vanishes instead of vorticity. In this case,we take the seven boundary conditions in (3.1)-(3.3) to be the same as in the previous case but in place of eighth condition (3.4), Happel boundary condition is used. Thus, vanishing of shear stress on the cell surface implies that

$$
T_{r \theta}^{(1)}(c, \theta)=0 .
$$

Applying the boundary conditions (3.1)-(3.3) with (4.6) and solving the resulting equations, we get the values of unknown constants appearing in (2.12) and (2.13). Therefore, we get the explicit expressions of the stream functions, and, hence, velocity distributions, pressure distributions, stress, vorticity, and the drag force may be evaluated. Instead, we report the values of the drag coefficient for the simpler cases as mentioned below. 


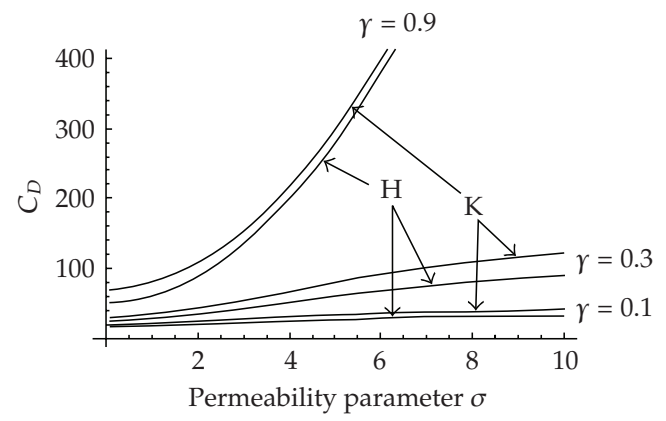

Figure 2: Variation of the drag coefficient $C_{D}$ versus permeability parameter $\sigma$ for various values of particle volume fraction $\gamma$.

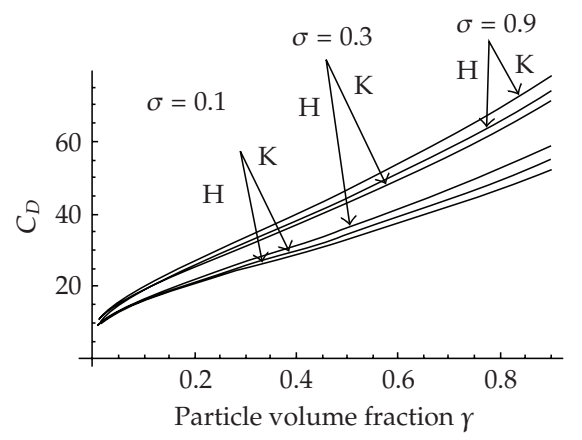

Figure 3: Variation of the drag coefficient $C_{D}$ versus particle volume fraction $\gamma$ for various values of permeability parameter $\sigma$.

\subsubsection{Drag on a porous circular cylinder in a cell}

In particular, when $a=0$, that is, $\ell=a / b=0$, then cylindrical shell will reduce to a porous circular cylinder of radius $b$. Thus, the value of the drag coefficient $C_{D}$ will come out as

$$
C_{D}=\frac{16 \pi \sigma^{2}\left[\sigma I_{1}(\sigma)\left(1+\gamma^{2}\right)-4 \gamma^{2} I_{2}(\sigma)\right]}{\operatorname{Re}\left[\sigma^{2}\left(\gamma^{2}-1\right)\left[\sigma I_{1}(\sigma)-2 I_{2}(\sigma)\right]+\left(8-\sigma^{2} \ln \gamma\right)\left[\sigma\left(1+\gamma^{2}\right) I_{1}(\sigma)-4 \gamma^{2} I_{2}(\sigma)\right]\right]} .
$$

\subsubsection{Drag on a solid cylinder in Happel cell model $(k \rightarrow 0)$}

Again, if permeability $k$ vanishes, that is, permeability parameter $\sigma \rightarrow \infty$, then the porous circular cylinder behaves like a solid cylinder of radius $b$. In this case, the value of the drag coefficient $C_{D}$ will become as

$$
C_{D}=\frac{16 \pi}{\left\{\operatorname{Re}\left[(\ln \gamma+1)-2 \gamma^{2} /\left(1+\gamma^{2}\right)\right]\right\}}
$$

which agrees with the result reported earlier by Happel [2] for the drag force experienced by a solid cylinder in a cell. 


\section{Conclusions}

Figure 2 shows that the comparison between Happel and Kuwabara results in the porous circular cylindrical shell for various values of the particle volume fraction $\gamma$, when permeability parameter $\sigma$ varies as parameter and $\ell=0.4$. It is seen that the variation of the drag coefficient $C_{D}$ is large in case of Kuwabara boundary condition in comparison to the case of Happel boundary condition. Figure 3 shows that the comparison between Happel and Kuwabara results in the porous circular cylinder for various values of the permeability parameter $\sigma$, when particle volume fraction $\gamma$ varies and $\ell=0.4$. It is seen that the variation of the drag coefficient $C_{D}$ is large in case of Kuwabara boundary condition in comparison to the case of Happel boundary condition when particle volume fraction $\gamma$ varies as parameter.

\section{Acknowledgments}

The first author is thankful to the Department of Science and Technology, Government of India for providing the financial assistance under its projects Grant no. SR/FTP/MS-07/2004 during this work. Authors acknowledge their sincere thanks to the reviewer for his valuable suggestions which led to much improvement in the presentation of the paper.

\section{References}

[1] J. Happel, "Viscous flow in multiparticle system: slow motion of fluids relative to beds of spherical particles," AIChE Journal, vol. 4, no. 2, pp. 197-201, 1958.

[2] J. Happel, "Viscous flow relative to arrays of cylinders," AIChE Journal, vol. 5, no. 2, pp. 174-177, 1959.

[3] S. Kuwabara, "The forces experienced by randomly distributed parallel circular cylinders or spheres in a viscous flow at small Reynolds numbers," Journal of the Physical Society of Japan, vol. 14, no. 4, pp. 527-532, 1959.

[4] I. Pop and P. Cheng, "Flow past a circular cylinder embedded in a porous medium based on the Brinkman model," International Journal of Engineering Science, vol. 30, no. 2, pp. 257-262, 1992.

[5] S. Datta and M. Shukla, "Drag on flow past a cylinder with slip," Bulletin of the Calcutta Mathematical Society, vol. 95, no. 1, pp. 63-72, 2003.

[6] G. Dassios and P. Vafeas, "The 3D Happel model for complete isotropic Stokes flow," International Journal of Mathematics and Mathematical Sciences, vol. 2004, no. 46, pp. 2429-2441, 2004.

[7] S. Deo, "Stokes flow past a swarm of porous circular cylinder with Happel and Kuwabara boundary conditions," Sadhana, vol. 29, no. 4, pp. 381-387, 2004.

[8] A. S. Kim and R. Yuan, "A new model for calculating specific resistance of aggregated colloidal cake layers in membrane filtration processes," Journal of Membrane Science, vol. 249, no. 1-2, pp. 89-101, 2005.

[9] G. Dassios, M. Hadjinicolaou, F. A. Coutelieris, and A. C. Payatakes, "Stokes flow in spheroidal particle-in-cell models with rappel and Kuwabara boundary conditions," International Journal of Engineering Science, vol. 33, no. 10, pp. 1465-1490, 1995.

[10] J. Happel and H. Brenner, Low Reynolds Number Hydrodynamics, Martinus Nijhoff, Hague, The Netherlands, 1983.

[11] H. C. Brinkman, "A calculation of the viscous force exerted by a flowing fluid on a dense swarm of particles," Applied Sciences Research, vol. A1, pp. 27-34, 1947.

[12] M. Abramowitz and I. A. Stegun, Handbook of Mathematical Functions, Dover, New York, NY, USA, 1970. 


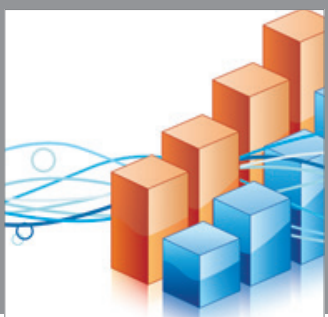

Advances in

Operations Research

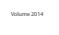

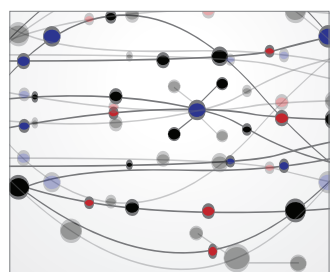

\section{The Scientific} World Journal
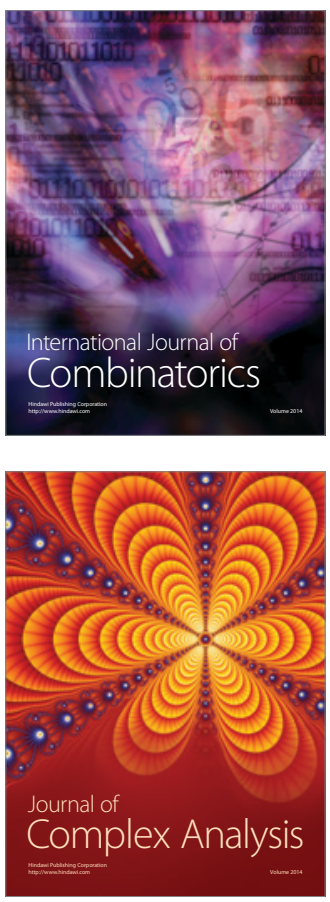

International Journal of

Mathematics and

Mathematical

Sciences
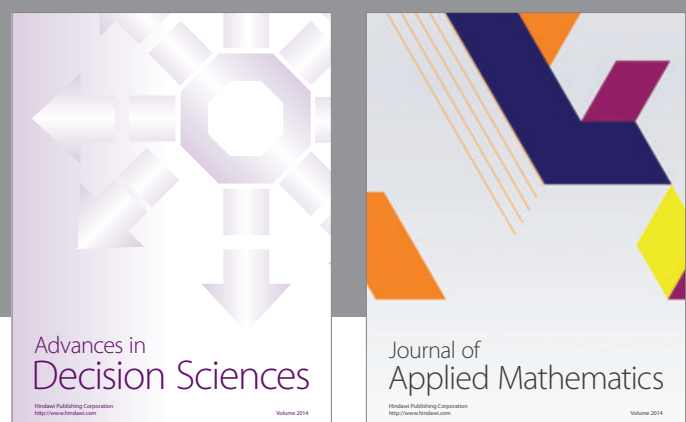

Journal of

Applied Mathematics
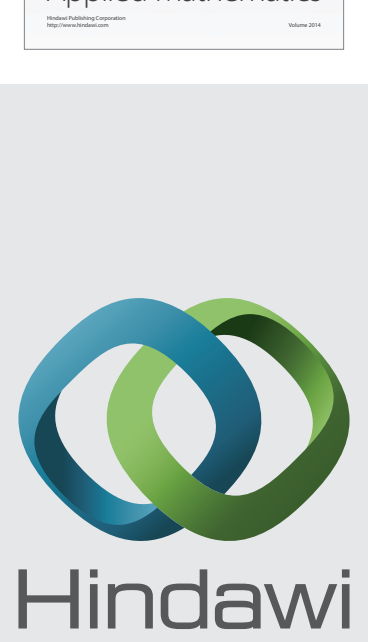

Submit your manuscripts at http://www.hindawi.com
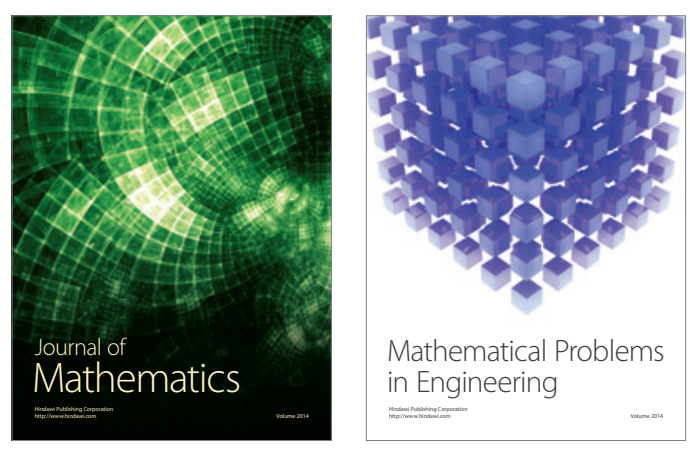

Mathematical Problems in Engineering
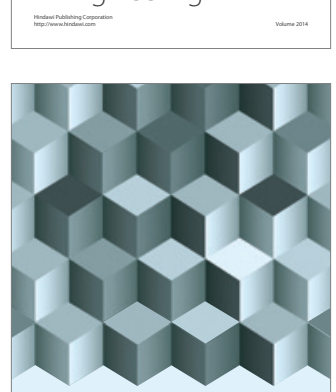

Journal of

Function Spaces
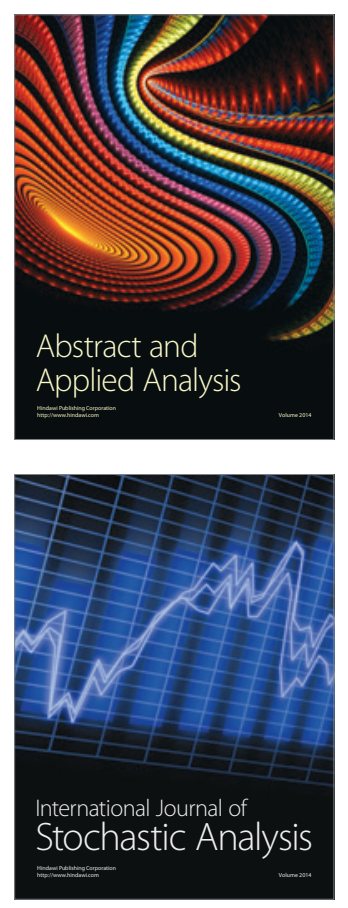

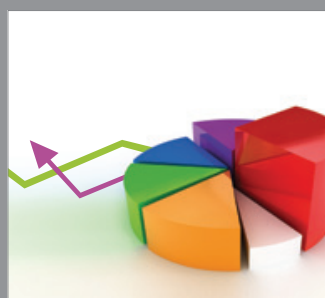

ournal of

Probability and Statistics

Promensencen
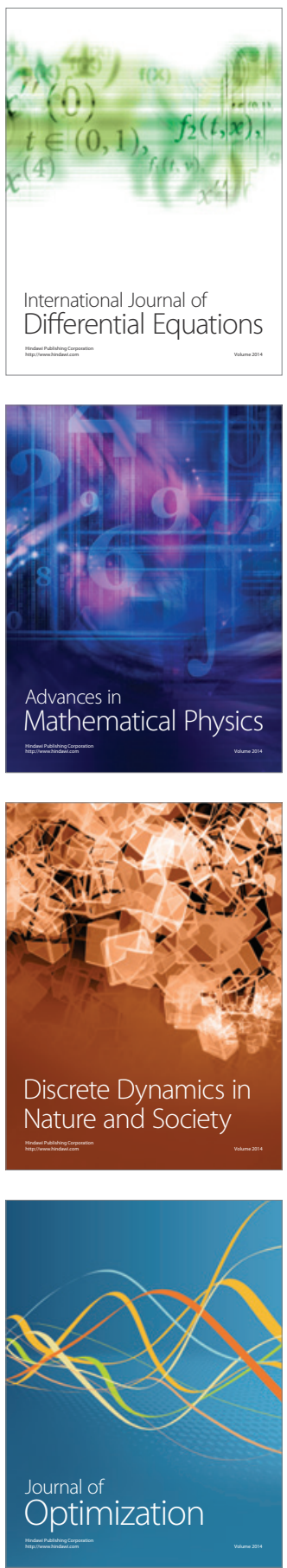